\title{
Investigations of Accelerated Climate Aged Wood Substrates by Fourier Transform Infrared Material Characterization
}

\author{
Bjørn Petter Jelle, ${ }^{1,2}$ Petra Rüither, ${ }^{1,2}$ and Per Jostein Hovde ${ }^{2}$ \\ ${ }^{1}$ Department of Materials and Structures, SINTEF Building and Infrastructure, 7465 Trondheim, Norway \\ ${ }^{2}$ Department of Civil and Transport Engineering, Norwegian University of Science and Technology (NTNU), 7491 Trondheim, Norway
}

Correspondence should be addressed to Bjørn Petter Jelle, bjorn.petter.jelle@sintef.no

Received 14 March 2012; Revised 19 June 2012; Accepted 20 June 2012

Academic Editor: John W. Gillespie

Copyright ( $\odot 2012$ Bjørn Petter Jelle et al. This is an open access article distributed under the Creative Commons Attribution License, which permits unrestricted use, distribution, and reproduction in any medium, provided the original work is properly cited.

Fourier transform infrared (FTIR) material characterization by applying the attenuated total reflectance (ATR) experimental technique represents a powerful measurement tool. The ATR technique may be applied on both solid state materials, liquids and gases with none or only minor sample preparations, also including materials which are nontransparent to infrared radiation. This facilitation is made possible by pressing the sample directly onto various crystals, for example, diamond, with high refractive indices, in a special reflectance setup. Materials undergoing ageing processes by natural and accelerated climate exposure, decomposition and formation of chemical bonds and products, may be studied in an ATR-FTIR analysis. In this work, the ATRFTIR technique is utilized to detect changes in selected wood building material substrates subjected to accelerated climate exposure conditions. Changes in specific FTIR absorbance peaks are designated to different wood deterioration processes. One aim is by ATR-FTIR analysis to be able to quantitatively determine the length of the wood ageing time before priming/treatment. Climate parameters like temperature (including freezing/thawing), relative air humidity, wind driven rain amount, solar and/or ultraviolet radiation, and exposure duration may be controlled in different climate ageing apparatuses. Both impregnated and raw wood samples have been employed in the experimental investigations.

\section{Introduction}

Wood deterioration of building materials due to natural climate ageing processes represents a problem. In natural climate ageing, the wood materials, for example, wood claddings, are exposed to several climate and climateinduced factors like

(i) solar radiation, that is, ultraviolet (UV), visible (VIS), and near infrared (NIR) radiation;

(ii) ambient infrared (IR) heat radiation (the resulting elevated temperature increases the rate of chemical degradation reactions, and also the rate of growth of rot and fungus up to limiting temperatures);

(iii) high and low temperatures;

(iv) temperature changes/cycles (relative temperature movements between different materials, number of freezing point passes during freezing/thawing); (v) water, for example, moisture, relative air humidity, rain (precipitation), and wind-driven rain;

(vi) physical strains, for example, snow loads;

(vii) wind;

(viii) erosion (also from above factors);

(ix) pollutions, for example, gases and particles in air;

(x) microorganisms;

(xi) oxygen;

(xii) time (determining the effect for all the factors above to work).

It should be noted that several and varying combinations of the climate exposure factors will occur, and the total climate strain may be substantially larger than the added sum of the single exposure factors. Also note that several microorgansims, for example, wood rot and mould fungus 
specifically, may not be listed as climate exposure factors. That is, for example, wood rot and mould fungus may in this context be viewed as (unwanted) results of the specific climate factors moisture and temperature for a certain exposure time with a sufficient supply of nourishment (e.g., wood). In fact, although commonly not regarded as so, the availability of fungal spores may be seen as a special climate exposure factor. Fungal spores are almost always present, except under sterile conditions. Finally, many microorganisms may also not be regarded as directly climate-induced exposure factors, and larger organisms like insects, birds, and animals do also represent potential wood degradation agents.

In this work, we are trying to utilize the attenuated total reflectance (ATR) Fourier transform infrared (FTIR) radiation technique in order to study wood decomposition in accelerated climate aged building material substrates. The ATR-FTIR technique makes it possible to study materials which are nontransparent to IR radiation in a pristine condition. That is, the extensive, time-consuming, and often cumbersome sample preparation by pressing thin $\mathrm{KBr}$ pellets as in traditional FTIR transmittance spectroscopy is avoided. This traditional technique might even change the sample material in question. The ATR technique is based on a special reflectance setup where the sample is pressed directly onto various crystals with high refractive indices, for example, diamond. One goal is to be able to quantitatively determine the wood decomposition by performing an ATR-FTIR analysis, including to determine the length of the wood ageing time before priming/treatment of the wood has to be done.

Experimentally, the task is challenging. Firstly, it may be difficult to differentiate the wood deterioration products on the attacked wood sample from the wood substrate material itself. Secondly, it may be complicated to be able to distinguish between various wood decomposition products. Thirdly, the wood material is not homogenous, which may complicate the measurements. Finally, with the ATR technique it is important to achieve and ensure a good contact with no air pockets between the sample and the ATR crystal in order to obtain correct quantitative results. With wood samples having relatively hard and rough surfaces such a good contact might be difficult to achieve and especially to ensure that the actual contact is the best one attainable. Several works are carried out applying infrared spectroscopy in studies of wood weathering and wood photodegradation, for example, by Anderson et al. [1, 2], Colom et al. [3], Humar et al. [4], Mohebby [5], Pandey and Pitman [6], Pandey [7], Sudiyani et al. [8], and Yamauchi et al. [9]. Anderson et al. [1,2] studied the effects of artificial weathering of softwoods and hardwoods with water alone, sunlight (xenon lamp) alone, and both water and sunlight applied in conjunction. ATR analyses were performed by Humar et al. [4] and Mohebby [5]. The common practice and most widely applied experimental method is the traditional $\mathrm{KBr}$ pellet sample technique in infrared transmittance modus. Although there exists many experimental challenges using the ATR-FTIR method on wood, this method is, nevertheless very promising and with many advantages and is therefore the subject of our investigations in this work. For application of the ATR-FTIR method on other building materials and processes, it is referred to the work by Jelle and Hovde [10] for FTIR investigations of wood rot decay and mould fungi growth on building materials and Jelle and Nilsen [11] for comparison of accelerated climate ageing methods of polymer building materials by FTIR. For a general review on accelerated climate ageing of building materials, components and structures in the laboratory, it is referred to the work of Jelle [12].

\section{Experimental}

2.1. Sample Materials. Altogether, nine different wood sample materials, five raw, non-impregnated and four impregnated wood samples, have been tested in various accelerated climate ageing apparatuses, in addition to natural outdoor weather ageing. In order not to overburden the reader, two representative samples have been chosen and discussed in the work presented here. The two wood samples are

sample A: Norway Spruce, Picea Abies L. Karst. (Ruptimal Surface, mechanically treated surface), measured dry mass density of $0.41 \mathrm{~kg} / \mathrm{dm}^{3}$. Raw, nonimpregnated sample;

sample H: pressure-treated Pine Class NP5 (chemically treated according to EN 351-1:2007), Pinus Sylvestris L., measured dry mass density of $0.50 \mathrm{~kg} / \mathrm{dm}^{3}$. Impregnated sample.

The small wood pieces needed for the FTIR measurements have been collected from the larger samples depicted in Figure 1.

2.2. Short Description of the Overall Study. The results presented here is part of a larger on-going study where several different wood samples are being subjected to both natural weather ageing and various accelerated climate ageing apparatuses, including comparisons between the natural and the artificial weathering methods. The various ageing apparatuses include an Atlas SC600 MHG Solar Simulator (see next section for details), a QUV apparatus weathering tester horizontal option with ponding and water spray (The Q-Panel Company, Cleveland, OH, USA), and a vertical accelerated climate simulator subjecting the sample area in turns to four different climate zones ( 1 hour in each climate zone), that is, one UV (UVA and UVB intensities are averaged to $14 \mathrm{~W} / \mathrm{m}^{2}$ and $1.4 \mathrm{~W} / \mathrm{m}^{2}$, respectively) and IR irradiation zone (black panel temperature of $63^{\circ} \mathrm{C}$ ), one water spray zone $\left(15 \mathrm{dm}^{3} /\left(\mathrm{m}^{2} \mathrm{~h}\right)\right)$, one freezing zone $\left(-20^{\circ} \mathrm{C}\right)$, and one indoor laboratory climate zone according to Nordtest Method NT Build 495 (2000). Again, in order to not overburden the reader, only selected results from the accelerated ageing in the Atlas Solar Simulator are shown, as the work presented here focuses on the FTIR material characterization of selected climate aged wood substrates and not on a comparison between various ageing methods.

2.3. Accelerated Ageing in Atlas Solar Simulator. The accelerated ageing was carried out in an Atlas SC600 MHG Solar Simulator climate chamber with a 2500 W MHG lamp. 


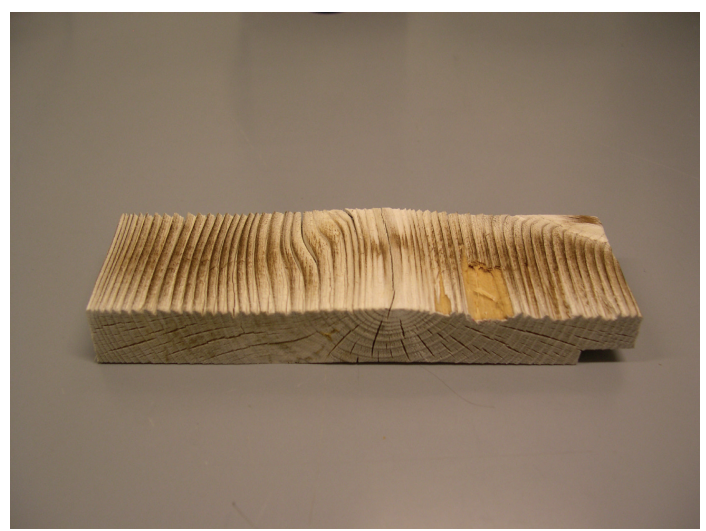

(a)

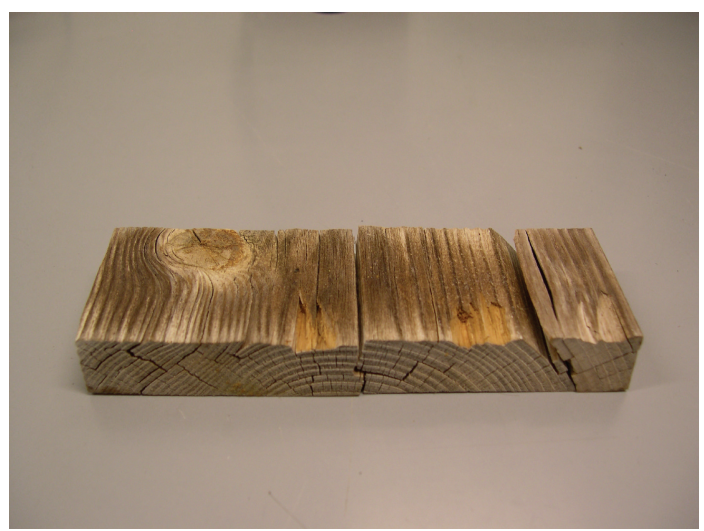

(b)

Figure 1: Wood sample A (a) and wood sample H (b) after 115 days of accelerated ageing in the Atlas Solar Simulator climate chamber. Direct solar radiation exposed side shown upwards. Small wood pieces are cut from the samples for use in the FTIR investigations.

The wood samples were tilted a bit from the horizontal $(8 \pm$ $\left.2^{\circ}\right)$ in order to let the water run off the surface. The samples were placed in the climate chamber with a distance of approximately $55 \mathrm{~cm}$ from the climate chamber glass ceiling to the samples, where the solar radiation intensity is reported to be $1200 \mathrm{~W} / \mathrm{m}^{2}$ at $100 \%$ lamp power intensity. During the ageing period, the UVA and UVB radiation intensity was measured at various times to be lying within the interval 60$80 \mathrm{~W} / \mathrm{m}^{2}$ and $3-6 \mathrm{~W} / \mathrm{m}^{2}$, respectively. The UV measurements were performed with a radiometer/photometer Model IL 1400A (International Light) with an UVA sensor and an UVB sensor. Note that a 6\% UVA fraction (like in solar radiation) of the total solar intensity $\left(1200 \mathrm{~W} / \mathrm{m}^{2}\right)$ yields $72 \mathrm{~W} / \mathrm{m}^{2}$ UVA radiation. The exposure duration consisted of 115 whole cycles of 24 hours, each cycle divided into 20 hours with a solar radiation intensity of $1200 \mathrm{~W} / \mathrm{m}^{2}$ and 4 hours with water spray application and no solar radiation exposure. The two water spray nozzles gave each $0.5 \mathrm{dm}^{3} / \mathrm{min}$, that is, $1 \mathrm{dm}^{3} / \mathrm{min}$ in total, which gives approximately $1.7 \mathrm{dm}^{3} /\left(\mathrm{m}^{2} \mathrm{~min}\right)$ assuming an even horizontal water distribution at the sample location in the whole climate chamber. Integrated up, the solar radiation (MHG lamp) energy is $24 \mathrm{kWh} / \mathrm{m}^{2}$ per day and $2760 \mathrm{kWh} / \mathrm{m}^{2}$ during 115 days. Likewise, assuming $70 \mathrm{~W} / \mathrm{m}^{2}$ as an average, the UVA radiation energy is $1.4 \mathrm{kWh} / \mathrm{m}^{2}$ per day and $161 \mathrm{kWh} / \mathrm{m}^{2}$ during 115 days. The temperature and relative air humidity were held constant at $63^{\circ} \mathrm{C}$ and $50 \% \mathrm{RH}$ during the solar radiation exposure and at $10^{\circ} \mathrm{C}$ and close to $100 \% \mathrm{RH}$ during the water spray application.

2.4. FTIR Measurements. The FTIR material characterization was carried out with a Thermo Nicolet 8700 FTIR spectrometer with a Smart Orbit accessory, that is, a horizontal attenuated total reflectance (ATR) accessory (single reflection) with a diamond crystal, in the wavelength range $4000 \mathrm{~cm}^{-1}(2.5 \mu \mathrm{m})$ to $400 \mathrm{~cm}^{-1}(25 \mu \mathrm{m})$ in an atmosphere with minimalized $\mathrm{CO}_{2}$ and $\mathrm{H}_{2} \mathrm{O}$ content through purging by a Parker Balston 74-5041 FTIR Purge Gas Generator. Each FTIR spectrum presented is based on a recording of 32 scans at a resolution of $4 \mathrm{~cm}^{-1}$. In order to ensure satisfactory contact between the ATR diamond crystal and the sample, three or more FTIR spectra were recorded at various locations on the sample. The wood surfaces are relatively hard, which complicates accurate quantitative measurements (height of absorbance peaks) due to varying contact with the ATR crystal for the different samples. Air between sample and ATR crystal results in a weaker absorbance signal. Unless other conditions indicate otherwise (e.g., inhomogeneities and impurities), the FTIR curves with the largest absorbance peaks represent the most correct measurements on one and the same sample with equal ageing time, and hence these curves are chosen as they are assumed to be the most correct ones. Qualitative measurements (location of absorbance peaks at wave numbers) do not represent a problem as long as the contact area is large enough to ensure a sufficient strong measurement signal. The FTIR spectra given in this work have not been ATR corrected, neither with respect to penetration depths nor absorbance band shifts, which both are dependent on the refractive indices of the sample and the ATR crystal (diamond in this case) and the angle of incident radiation. The penetration depth is in addition also dependent on the radiation wavelength and increases with increasing wavelength (decreasing wave number), that is, non-corrected ATR spectra have much stronger absorbance bands at longer wavelengths (smaller wave numbers) than at shorter wavelengths (larger wave numbers). Note that it should always be stated if an ATR-FTIR spectrum has been ATR corrected or not, for example, important during computerized database spectra comparison searches. As we in this work are solely comparing the ATR-FTIR spectra measured within this work, there is no need for performing any ATR corrections. Besides, the raw ATR-FTIR data in either transmittance or logarithmic absorbance mode is usually preferred. It should also be noted that one often do not know the refractive indices of the samples which are measured, thus errors might be introduced in the ATR corrected spectra as the refractive index of the sample is an input parameter in the ATR correction. Figure 2 shows a wood piece from sample 


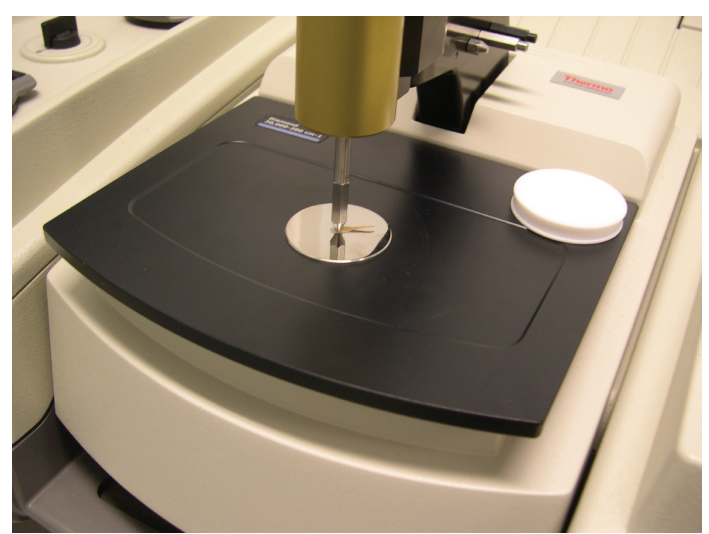

(a)

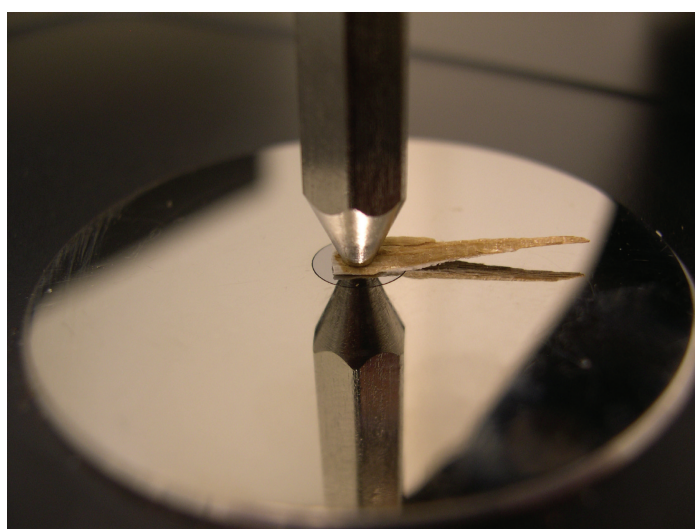

(b)

FIgURe 2: A wood piece from sample A was pressed onto the diamond crystal in the ATR accessory connected to the FTIR spectrometer. In the close-up photo to the right, the lower steel pin is only a reflection of the above steel pin in the polished mirror-like sample plate.

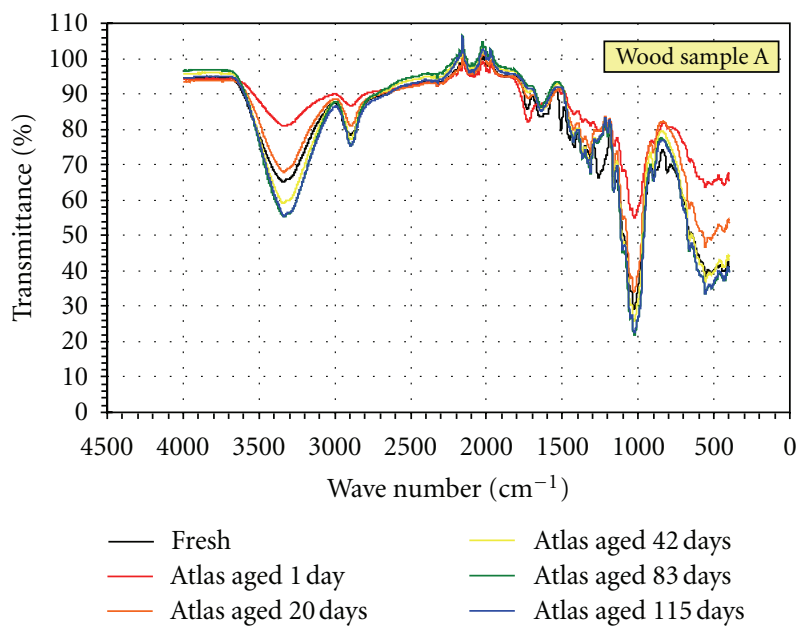

FIGURE 3: Transmittance versus wave number between 4000$400 \mathrm{~cm}^{-1}$ for wood sample A during accelerated ageing in an Atlas Solar Simulator.

A pressed onto the diamond crystal in the ATR accessory connected to the FTIR spectrometer.

\section{Results and Discussion}

FTIR transmittance spectra versus wave number between $4000-400 \mathrm{~cm}^{-1}$ for the sample A and sample $\mathrm{H}$ at various accelerated ageing levels are presented in Figures 3 and 4. Further close-ups of these spectra are shown as FTIR absorbance versus wave number between $1900-700 \mathrm{~cm}^{-1}$ in Figures 5 and 6 . Finally, as a reference to avoid any misinterpretations, the distilled water FTIR transmittance spectrum is plotted together with the fresh and aged 115 days sample A spectra in Figure 7. Note that the irregularities in the FTIR spectra between $2200-1900 \mathrm{~cm}^{-1}$ (Figures 3 and 4) are due to the very large absorption in the ATR diamond crystal between these wave numbers, which represents the

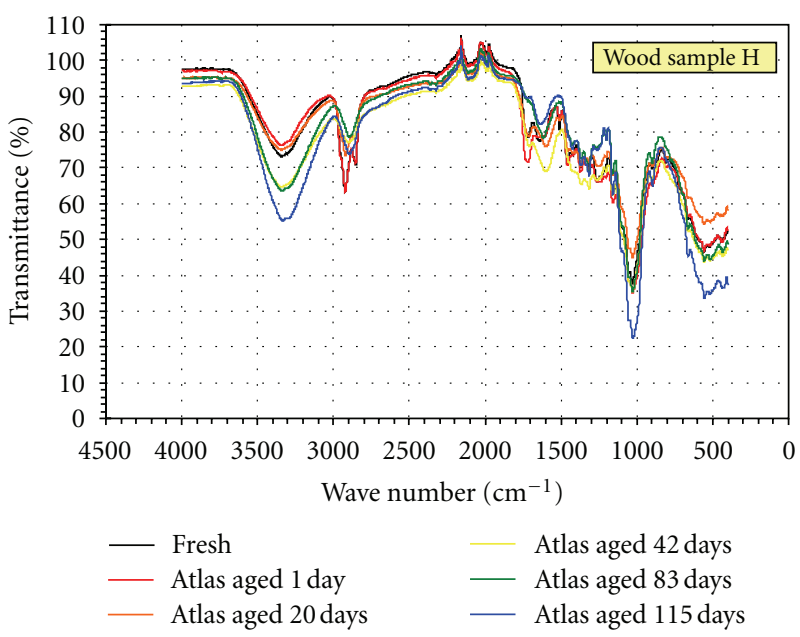

FIgURE 4: Transmittance versus wave number between 4000$400 \mathrm{~cm}^{-1}$ for wood sample $\mathrm{H}$ during accelerated ageing in an Atlas Solar Simulator.

weak point in an otherwise excellent material choice for ATR applications.

As the absorption of electromagnetic radiation, for example, IR radiation, follows the Beer-Lambert law, that is, the radiation is decreasing exponentially with the penetration depth in the actual material, it is often helpful to plot the spectra on a logarithmic absorbance scale versus wavelength. Hence, a representative spectrum is chosen from each of the samples and plotted on a logarithmic absorbance scale for quantitative studies. Mathematically and physically, it follows that a doubling of the logarithmic absorbance, also called optical density, is interpreted as a doubling of material thickness or a doubling of concentration of absorption active agents.

The wood sample material undergoes chemical changes and may then as a result of the chemical reactions change the actual thickness of the sample. In this work, the experiments are conducted by applying the ATR equipment with the FTIR 


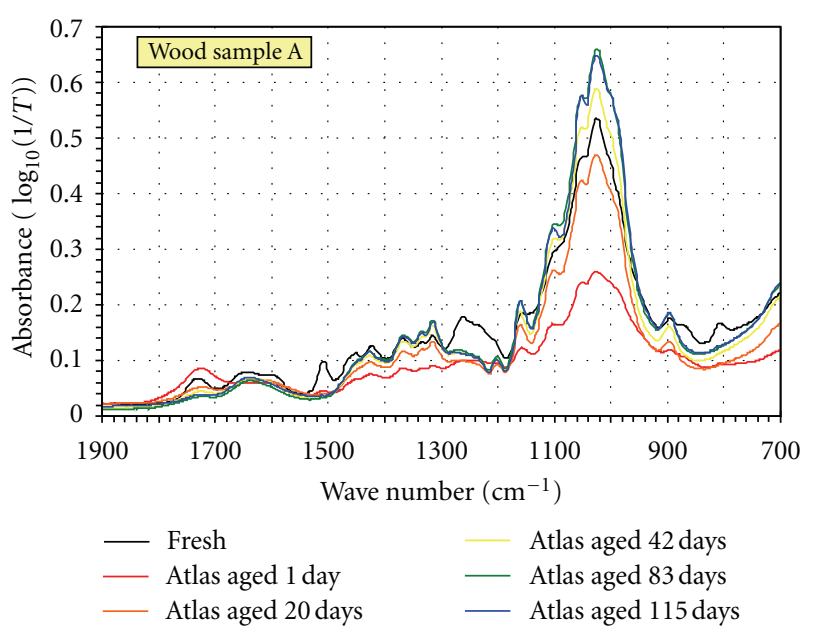

Figure 5: Absorbance (logarithmic) versus wave number between $1900-700 \mathrm{~cm}^{-1}$ for wood sample A during accelerated ageing in an Atlas Solar Simulator.

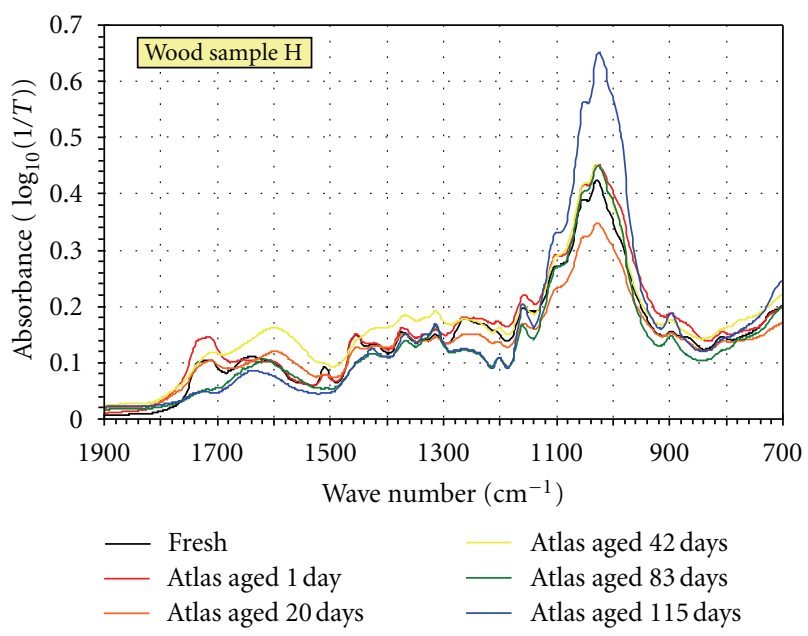

FIgURE 6: Absorbance (logarithmic) versus wave number between $1900-700 \mathrm{~cm}^{-1}$ for wood sample $\mathrm{H}$ during accelerated ageing in an Atlas Solar Simulator.

spectrometer. Hence, the IR radiation is only penetrating into a thin surface layer of the actual sample. With respect to the experiments carried out in this work, the material thickness will then be regarded as approximately constant, that is, the change in the IR absorbance is explained by an increase or decrease of absorption active species within the sample material undergoing the chemical transformation.

By inspecting the FTIR spectra (Figures 3-6) more closely, it is found that several absorbance peaks in the fresh, non-aged wood samples (both $\mathrm{A}$ and $\mathrm{H}$ ) are diminished and finally vanished during the accelerated ageing in the Atlas Solar Simulator. These absorbance peaks are located around $1730-1710 \mathrm{~cm}^{-1}$ (varying with different wood specimens), $1510 \mathrm{~cm}^{-1}, 1260 \mathrm{~cm}^{-1}$, and $810 \mathrm{~cm}^{-1}$. The peak around $1730-1710 \mathrm{~cm}^{-1}$ is attributed to carbonyl $(\mathrm{C}=\mathrm{O})$ stretching in hemicellulose, whereas the peak around $1510 \mathrm{~cm}^{-1}$ arises from the $\mathrm{C}=\mathrm{C}$ stretching of the aromatic ring in lignin. These

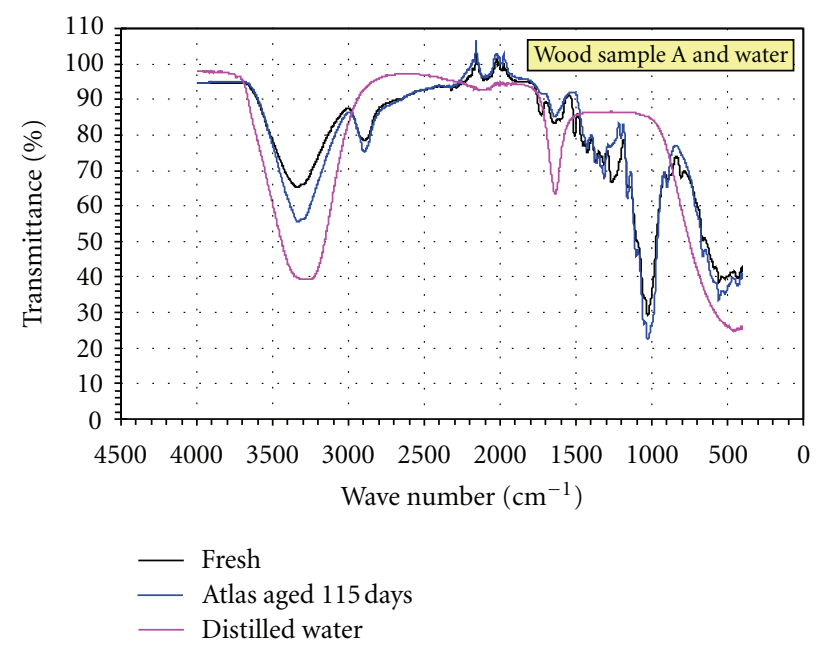

FIgURE 7: Transmittance versus wave number between 4000$400 \mathrm{~cm}^{-1}$ for wood sample A during accelerated ageing (fresh and 115 days, see Figure 3 for more details) in an Atlas Solar Simulator including a comparison with the distilled water spectrum.

two peaks, located around $1730-1710 \mathrm{~cm}^{-1}$, and $1510 \mathrm{~cm}^{-1}$ represent good candidates for studying wood decomposition by climate ageing through FTIR analysis.

In addition, for wood sample $\mathrm{H}$ (impregnated sample), the two sharp and distinct characteristic absorbance peaks between $3000-2800 \mathrm{~cm}^{-1}$ (CH stretch, which may be attributed to the impregnation agent) disappear after some ageing time. The fresh, non-aged wood sample A does not have these two distinct absorbance peaks, only a broad peak in the same wavelength range (similar to the aged peak for sample $\mathrm{H})$.

There are some other changes (i.e., increase and/or decrease) in the absorbance peaks during the ageing period also, but generally no complete disappearance of peaks and neither appearance of new peaks. Note that the water absorbance peaks are located at $3265 \mathrm{~cm}^{-1}$ (broader peak: $-\mathrm{OH}$ ) and $1637 \mathrm{~cm}^{-1}$ (narrower peak: -O-), and changes in the FTIR spectra around these wave numbers might also be due to various moisture levels in the samples. Also note that different adjacent neighbour atoms in a compound, for example, in a polymer chain, will shift the wave number somewhat for the absorbance peak corresponding to the chemical bond in question.

Hence, in this study it has been demonstrated that the ATR-FTIR spectroscopical technique may be applied in order to detect different levels of climate induced wood decomposition or ageing. Further studies and experimental investigations are needed in order to elaborate the suitability, the reliability, the limitations, and the future potential possibilities of this method. In future applications of this experimental method as a detection tool capable of differentiating between various wood decomposition or ageing levels, there is a need to build up large reference databases containing FTIR spectra of the different species at selected ageing levels. 


\section{Conclusions}

This study has demonstrated that the attenuated total reflectance (ATR) Fourier transform infrared (FTIR) spectroscopical technique may be applied in order to detect different levels of climate induced wood decomposition or ageing. However, further studies and experimental investigations are needed in order to elaborate the suitability, the reliability, the limitations, and the future potential possibilities of this method.

\section{Acknowledgments}

This work has been supported by the Research Council of Norway, AF Gruppen, Glava, Hunton Fiber as, Icopal, Isola, Jackon, maxit, Moelven ByggModul, Rambøll, Skanska, Statsbygg and Takprodusentenes forskningsgruppe through the SINTEF and NTNU research project Robust Envelope Construction Details for Buildings of the 21st Century (ROBUST).

\section{References}

[1] E. L. Anderson, Z. Pawlak, N. L. Owen, and W. C. Feist, "Infrared studies of wood weathering. Part I: softwoods," Applied Spectroscopy, vol. 45, pp. 641-647, 1991.

[2] E. L. Anderson, Z. Pawlak, N. L. Owen, and W. C. Feist, "Infrared studies of wood weathering. Part II: hardwoods," Applied Spectroscopy, vol. 45, pp. 648-652, 1991.

[3] X. Colom, F. Carrillo, F. Nogués, and P. Garriga, "Structural analysis of photodegraded wood by means of FTIR spectroscopy," Polymer Degradation and Stability, vol. 80, no. 3, pp. 543-549, 2003.

[4] M. Humar, B. Bučar, and F. Pohleven, "Brown-rot decay of copper-impregnated wood," International Biodeterioration and Biodegradation, vol. 58, no. 1, pp. 9-14, 2006.

[5] B. Mohebby, "Attenuated total reflection infrared spectroscopy of white-rot decayed beech wood," International Biodeterioration and Biodegradation, vol. 55, no. 4, pp. 247-251, 2005.

[6] K. K. Pandey and A. J. Pitman, "FTIR studies of the changes in wood chemistry following decay by brown-rot and white-rot fungi," International Biodeterioration and Biodegradation, vol. 52, no. 3, pp. 151-160, 2003.

[7] K. K. Pandey, "Study of the effect of photo-irradiation on the surface chemistry of wood," Polymer Degradation and Stability, vol. 90, no. 1, pp. 9-20, 2005.

[8] Y. Sudiyani, Y. Imamura, S. Doi, and S. Yamauchi, "Infrared spectroscopic investigations of weathering effects on the surface of tropical wood," Journal of Wood Science, vol. 49, no. 1, pp. 86-92, 2003.

[9] S. Yamauchi, Y. Sudiyani, Y. Imamura, and S. Doi, "Depth profiling of weathered tropical wood using Fourier transform infrared photoacoustic spectroscopy," Journal of Wood Science, vol. 50, no. 5, pp. 433-438, 2004.

[10] B. P. Jelle and P. J. Hovde, "Fourier transform infrared radiation spectroscopy applied for wood rot decay and mould fungi growth detection," Advances in Materials Science and Engineering. In press.

[11] B. P. Jelle and T. N. Nilsen, "Comparison of accelerated climate ageing methods of polymer building materials by attenuated total reflectance Fourier transform infrared radiation spectroscopy," Construction and Building Materials, vol. 25, no. 4, pp. 2122-2132, 2011.

[12] B. P. Jelle, "Accelerated climate ageing of building materials, components and structures in the laboratory," Journal of Materials Science, vol. 47, no. 18, pp. 6475-6496, 2012. 

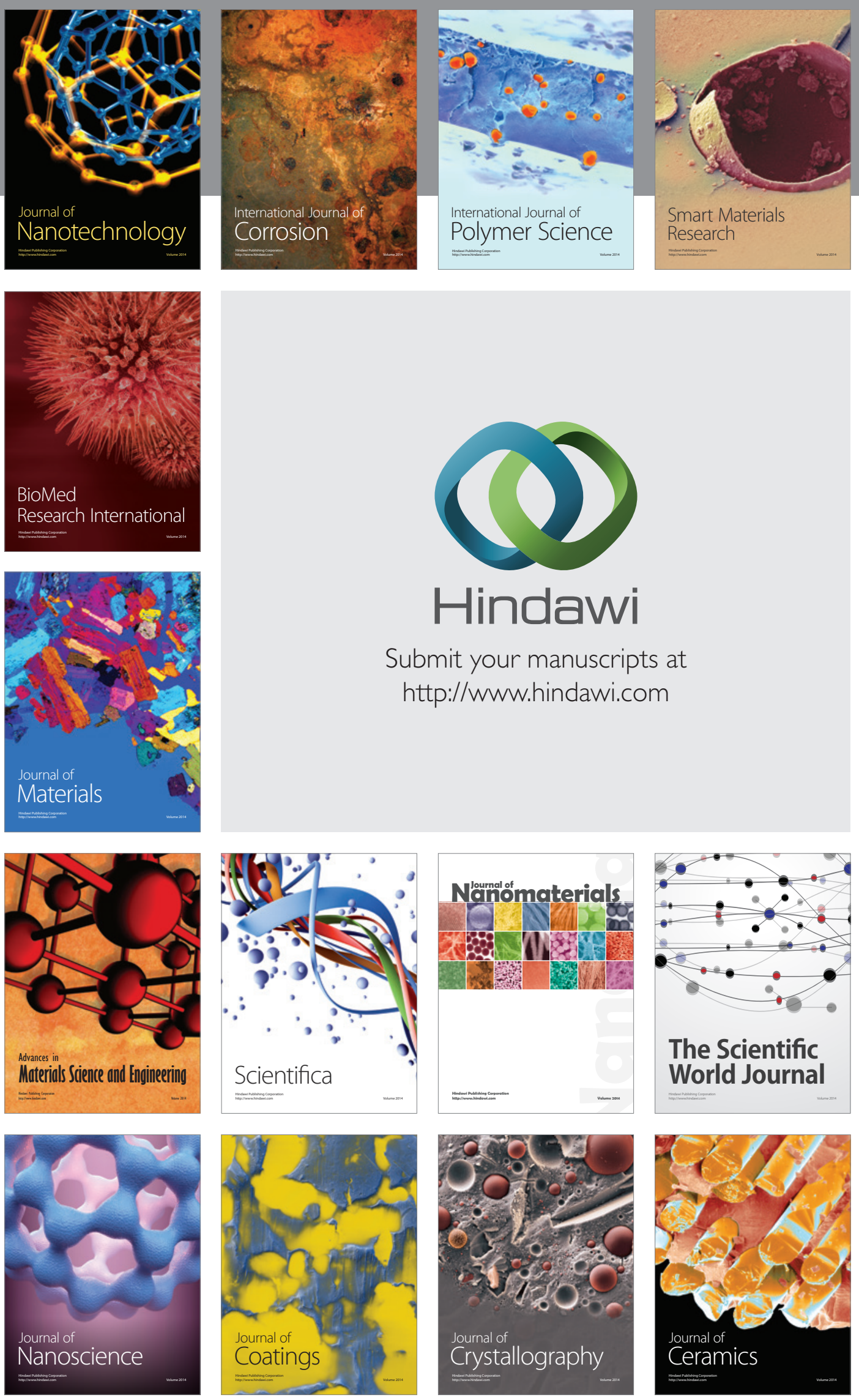

The Scientific World Journal

Submit your manuscripts at

http://www.hindawi.com

\section{World Journal}

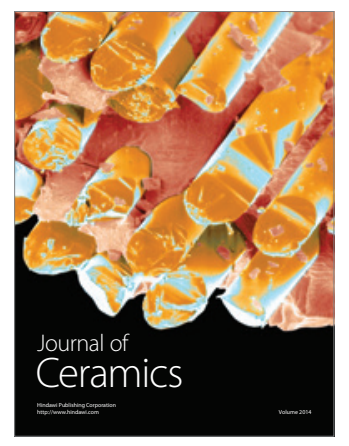

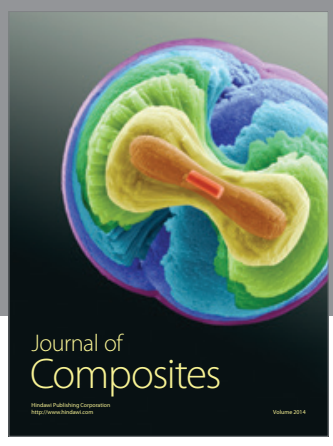
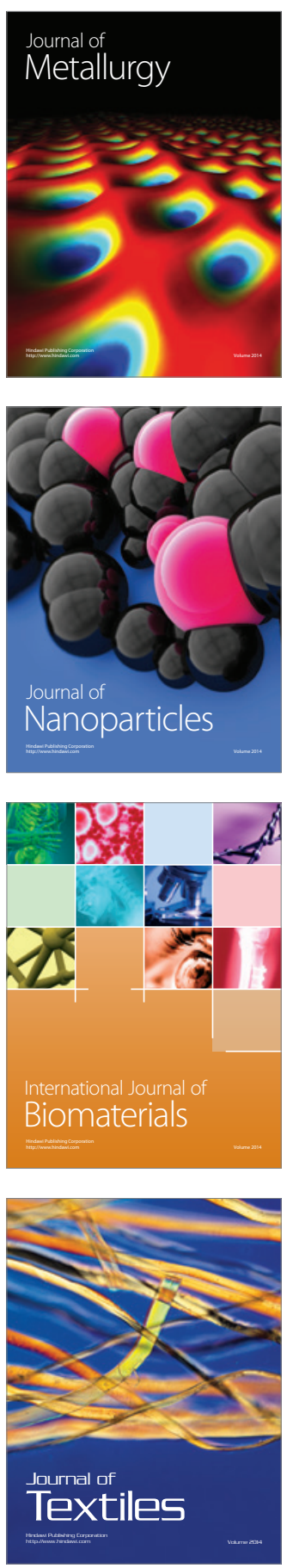